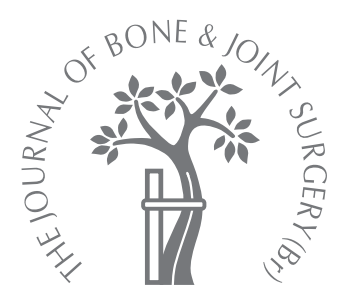

\title{
The relationship of the medially-offset stem of the tibial component to the medial tibial cortex in total knee replacements in Korean patients
}
J. H. Yoo,
Y. G. Kang,
C. B. Chang,
S. C. Seong,
T. K. Kim

From the Seoul

National University

Bundang Hospital,

Seoul, Korea

\begin{abstract}
We examined the placement of the stem in relation to the medial tibial cortex when using total knee replacements (TKRs) with medially-offset tibial stems in Korean patients. Measurements were performed on the pre- and post-operative radiographs of 246 osteoarthritic knees replaced between January 2005 and May 2006 using the Genesis II or E-motion TKR with a medially-offset stem. Pre-operatively, we measured the distance between the mechanical axis and that of the tibial shaft and post-operatively, that between the midline of the tibial stem and the axis of the shaft.

Knees were identified in which there was radiological contact between the tip of the stem and the medial tibial cortex. The mechanical axis was located medial to the axis of the shaft in 203 knees (82.5\%). Post-operatively, the midline of the tibial stem was located medial to the tibial shaft axis in 196 knees $(79.7 \%)$. In 16 knees $(6.5 \%)$ there was radiological contact between the tibial stem or cement mantle and the medial tibial cortex.

Our study has shown that the medially-offset stem in the tibial component may not be a good option for knees undergoing replacement for advanced osteoarthritis in some Korean patients.
\end{abstract}

\footnotetext{
J. H. Yoo, MD, Orthopaedic Surgeon

Department of Orthopaedic Surgery

National Police Hospital, 58 Garak-dong Street, Songpa-gu, Seoul 138-708, Korea.

Y. G. Kang, BSc, Clinical Investigator

C. B. Chang, MD, PhD, Assistant Professor

T. K. Kim, MD, PhD, Assistant Professor

Joint Reconstruction Centre Seoul National University Bundang Hospital, 300 Gumidong, Bundang-gu, Seongnamsi, Gyeonnggi-do, 464-707, Korea.

S. C. Seong, MD, PhD, Professor

Department of Orthopaedic Surgery

Seoul National University Hospital, 28 Yeongon-dong Chongno-gu, Seoul 110-744, Korea.

Correspondence should be sent to Professor T. K. Kim; e-mail: osktk@snubh.org
}

(C)2008 British Editorial Society of Bone and Joint Surgery doi:10.1302/0301-620X.90B1. $19605 \$ 2.00$

$J$ Bone Joint Surg $[\mathrm{Br}]$ 2008;90-B:31-6.

Received 26 April 2007; Accepted after revision 21 August 2007
The stem of the tibial component in a total knee replacement (TKR) is designed to prevent subsidence and loosening and has an important role in load-bearing and stress protection for the proximal tibia. ${ }^{1-5}$ The optimal performance of the stem relies on the proper placement of the tibial component. The tibial tray must be positioned so that it covers the maximum area of the prepared bone surface without overhang. The stem is placed parallel to the mechanical axis of the tibia and centered within the medullary canal to avoid impingement against the tibial cortex..$^{2,6-9}$ Mismatch between the position of the stem and the axis of the shaft may produce poor cover of the proximal tibia, and result in migration of the component, an uneven cement mantle and malalignment of the limb. $6,7,10,11$ These problems may be more marked in revision TKR, in which the tibial stem is generally longer. ${ }^{12}$

In order to avoid mismatch between the position of the stem and the axis of the tibia, components need to be designed to accommodate the anatomical features. While most of the current TKR systems have a tibial component with a symmetrically-shaped and centrally-positioned stem, some have a stem with a medial offset on an asymmetrical baseplate. ${ }^{7,13,14}$ These modified designs have been based on anatomical studies in Caucasian subjects in whom the medullary canal of the tibia is biased medially from the centre of the tibial plateau. .,13,14 $^{-1}$

However, several studies have suggested that in Asian subjects, the anatomical features of the proximal tibia may be different from those of Caucasians. ${ }^{10,15-17}$ In our practice for Korean patients undergoing TKR, we have noticed during pre-operative radiological planning that the mechanical axis was located medially to the axis of the shaft in many patients. When TKR was performed using components with a medially-offset tibial stem, we frequently experienced contact between the tip of the stem and the medial cortex of the tibia, which sometimes forced us to insert a smaller tibial component. We have attempted to determine whether the medially-offset stem in a tibial component would fit the anatomy of the tibia in Korean patients undergoing TKR for advanced osteoarthritis (OA). Our hypothesis was that the axis of the tibial shaft would be located more laterally than the mechanical axis of the tibia so that the medially-offset stem would not be suitable for such patients.

\section{Patients and Methods}

Between January 2005 and May 2006, 167 Korean patients (246 knees) with OA were included in the study. Any patient with a pre- 
Table I. Comparisons of the medial offset stem between the Genesis II and E-motion implants

\begin{tabular}{llll}
\hline \multirow{2}{*}{ Implant size } & \multicolumn{2}{l}{ Genesis II/E-motion } \\
\cline { 2 - 4 } & Width of tibial tray $(\mathbf{m m})$ & Amount of medial offset $(\mathbf{m m})$ & Proportion of medial offset to the width (\%) \\
\hline 1 & $60 / 59$ & $0.8 / 2.0$ & $1.3 / 3.4$ \\
2 & $64 / 63$ & $0.8 / 2.0$ & $1.2 / 3.2$ \\
3 & $68 / 67$ & $1.5 / 2.0$ & $2.2 / 3.0$ \\
4 & $71 / 71$ & $1.5 / 2.5$ & $2.1 / 3.5$ \\
5 & $74 / 75$ & $2.3 / 2.5$ & $3.0 / 3.3$ \\
6 & $77 / 79$ & $2.3 / 2.5$ & $2.9 / 3.2$ \\
7 & $81 / 83$ & $3.0 / 3.0$ & $3.7 / 3.6$ \\
8 & $85 / 87$ & $3.0 / 3.0$ & $3.5 / 3.5$ \\
\hline
\end{tabular}

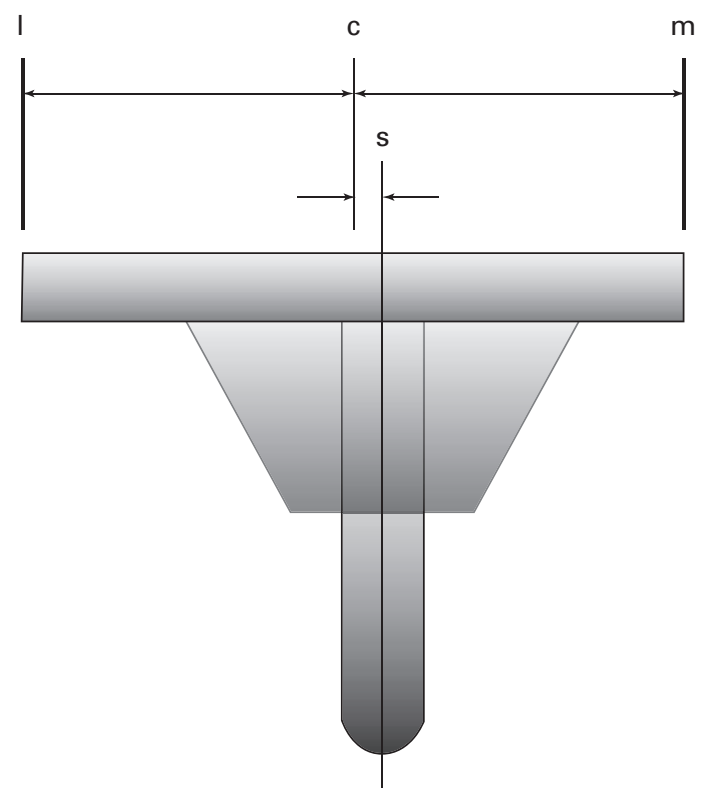

Fig. 1

Diagram showing a right tibial component with a medially-offset stem The tip of the stem (s) is located medial to the centre of the tibial tray (c) which is equidistant from the medial $(\mathrm{m})$ and lateral $(\mathrm{I})$ borders of the tibial component. The distance between $\mathrm{c}$ and $\mathrm{s}$ between the black arrows corresponds to the amount of medial offset.

existing abnormality affecting the alignment of the leg such as a previous fracture, deformity, or congenital anomaly was excluded. In the 138 women, (205 knees) the mean age was 68.3 years (50 to 83 ), the mean height was $151.6 \mathrm{~cm}$ (138 to 168), the mean weight $62 \mathrm{~kg}$ (42 to 98) and the mean body mass index (BMI) $26.9 \mathrm{~kg} / \mathrm{m}^{2}$ (19 to 41$)$. In the 29 men (41 knees) the mean age was 70.6 years ( 59 to 82 ), the mean height $162.7 \mathrm{~cm}$ (153 to 174), the mean weight $67 \mathrm{~kg}$ (51 to 82) and the mean BMI $25.3 \mathrm{~kg} / \mathrm{m}^{2}$ (21 to 30 ). Informed consent was obtained from each patient and ethical approval granted by the institutional review board of our hospital for the study.

A Genesis II TKR (Smith \& Nephew, Memphis, Tennessee) was implanted in 125 knees and the E-motion
TKR (B. Braun-Aesculap, Tuttlingen, Germany) in 121 knees. The former has a fixed-bearing insert while the E-motion implant has a mobile-bearing, but both have the design feature of the medially-offset stem with an asymmetrical tibial tray (Table I; Fig. 1). The extent of the medial offset varies with the designs and sizes of the implant but, overall, components of the E-motion system have a greater medial offset than those of the Genesis II system. The diameter of the tibial stem of the Genesis II TKR is $9 \mathrm{~mm}$ and in the E-motion TKR it is $\geq 12 \mathrm{~mm}$.

All the operations were performed by a single surgeon (TKK) using a standard operative technique comprising a medial parapatellar arthrotomy and femoral and tibial bone resections which were conducted using intramedullary and extramedullary guides, respectively. In performing resections of tibial bone, the intention was to produce a surface perpendicular to the mechanical axis of the tibia defined as the line connecting the midpoint between the medial and lateral intercondylar eminences of the tibia and the midpoint of the dome of the talus. After the size of the tibial component had been selected, it was placed as laterally as possible to improve the patellofemoral tracking. In the Genesis II knees, the rotational alignment of a tibial component was planned with reference to the medial one-third of the tibial tuberosity and confirmed by checking the tibiofemoral movement and patellofemoral tracking with trial components. In the E-motion knees, which allowed passive rotational adjustment through the mobile polyethylene insert, the tibial component was placed so that the prepared bone surface was covered maximally. Care was taken to avoid any medial overhang of the tibial tray when choosing the size and placing the component in either design. In all cases, the patella was resurfaced and fixation of the implant was carried out with cement.

Radiological measurements were performed using a full-length weight-bearing anteroposterior (AP) radiograph of the leg including the hip, knee and ankle. All the radiographs were taken with the patients in the twolegged stance position. The tibial shaft axis was defined as the line connecting the centre of the intramedullary canal $10 \mathrm{~cm}$ distal to the knee and $10 \mathrm{~cm}$ proximal to the ankle. 


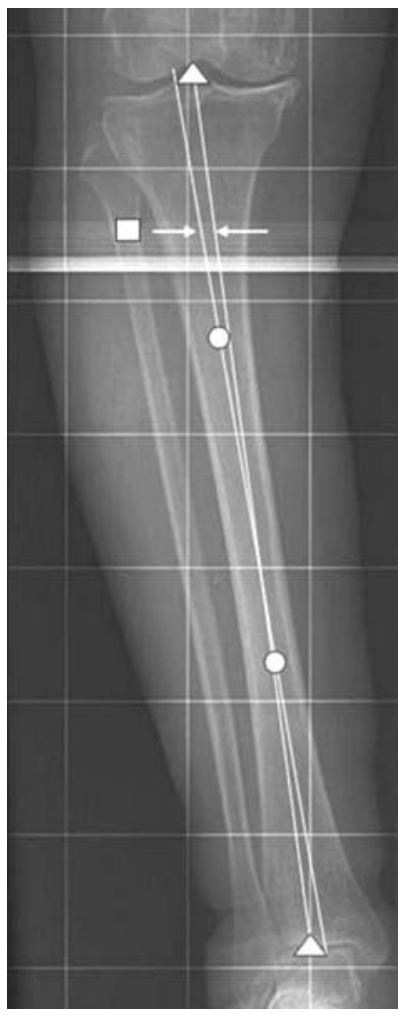

Fig. 2a

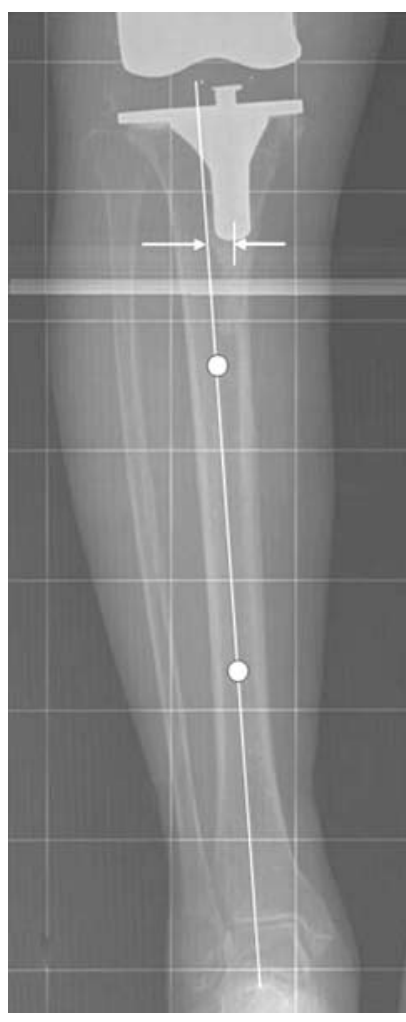

Fig. $2 b$
Figure $2 \mathrm{a}$ - Anteroposterior pre-operative radiograph showing the angle and distance between the mechanical axis (white triangles) and the tibial shaft axis (white circles). The distance (white arrows) was measured at a site $5 \mathrm{~cm}$ distal to the tip of the proximal fibula (white box). Figure $2 \mathrm{~b}-$ Anteroposterior post-operative radiograph showing the distance between the tip of the stem and the tibial shaft axis measured at the level of the tip (between the white arrows).

Measurements of the pre-operative radiographs included the angle between the mechanical axis and the axis of the tibial shaft and the distance separating the mechanical and the shaft axes at a site $5 \mathrm{~cm}$ distal to the tip of the proximal fibula (Fig. 2a). A negative value was ascribed to this measurement if the mechanical axis was located medial to the axis of the shaft and a positive value if it was located lateral to it. The proportion of the knees which had the mechanical axis medial to the tibial shaft axis was calculated.

In the post-operative radiographs, the distance between the tip of the stem and the axis of the shaft at the level of tip of the stem was measured (Fig. 2b). A negative value was used to denote the tip of the stem medial to the axis and a positive value when it was placed laterally. The proportion of the knees with the tip medial to the axis was calculated. In addition, cases of radiological evidence of contact between the distal end of the stem and the inner margin of the medial tibial cortex were identified. Contact was defined as apposition between the cement mantle and the cortex (Fig. 3a) or the presence of direct contact of the stem with the medial cortex (Fig. 3b). When the contact between the cement mantle and the medial cortex was found, only cases in which the medial side of the cement mantle was thinner than the lateral side by more than $1 \mathrm{~mm}$ were counted as knees with radiological contact.

To assess intra- and interobserver reliabilities of all measurements, 15 knees were randomly chosen from the series and three different investigators (JHY, YGK, TKK) examined them on two separate occasions. The second evaluations were performed after an interval of two weeks and the investigators were blinded to the results of the first evaluation. Intra- and interobserver reliabilities were assessed using the intraclass correlation coefficient which can assume any value between 0 and 1 , when a higher value means better agreement. A typical interpretation of the intraclass correlation coefficient values is that those values greater than 0.75 represent good agreement and those smaller than 0.4 poor agreement. ${ }^{18,19}$ In our study, intraclass correlation coefficient values of all measurements were greater than 0.9 both for intra- and interobserver reliabilities. Since the assessments of reliability validated the satisfactory intra- and interobserver reliabilities, one investigator (YGK) performed the radiological measurements for all cases.

Statistical analysis. This was performed using the SPSS version 12.0 software (SPSS Inc., Chicago, Illinois) and a p-value $<0.05$ was considered to be significant. The associations between the demographic factors (age, height, weight, BMI) and the pre-operative radiological parameters (the angle and distance between the mechanical and tibial shaft axes) were tested using correlation analyses (Pearson's coefficient). The differences in the pre-operative parameters by gender were evaluated using the Student's $t$-test. The associations of the pre-operative parameters with the occurrence of radiological contact between the tibial stem and the medial cortex of the tibia were studied using logistic regression analysis. Since contact could be influenced by the coronal and sagittal alignments of the tibial component, this was performed with the coronal and sagittal alignment controlled. The coronal and sagittal alignments of the tibial component were represented by the medial angle created between the tibial plate and a line connecting the midpoint of the tibial tray and the centre of the talus and the angle of the posterior slope of the tibial component, respectively.

Comparative analyses between knees with Genesis II and those with E-motion implants were made for the parameters of pre-operative and post-operative measurements. Statistical significance was determined by Student's $t$-test for the differences in the means of the angles and the distances between the mechanical and tibial shaft axes. The chi-squared test was used for comparisons of the proportions of cases in which the mechanical axis was located medial to the tibial shaft axis, those in which the tip of the stem was located medial to this axis and those in which there was radiological contact between the tip of the stem and the medial cortex of the tibia. 


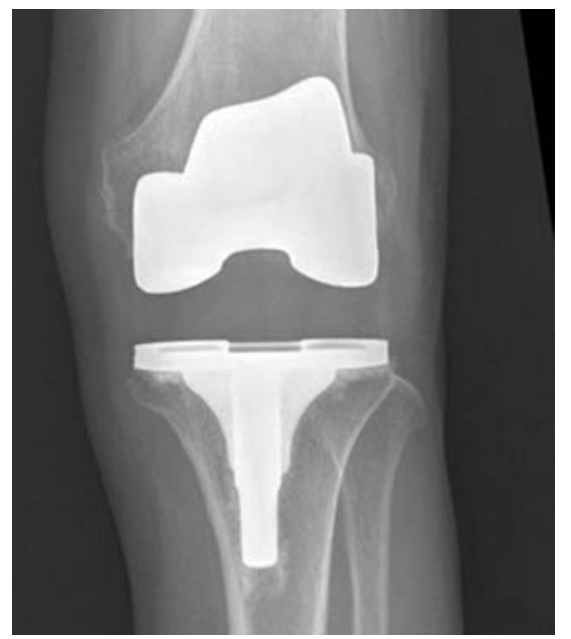

Fig. 3a

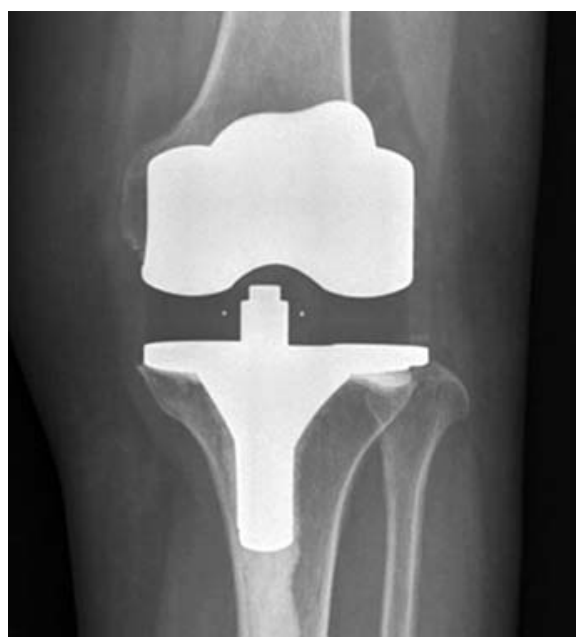

Fig. $3 b$

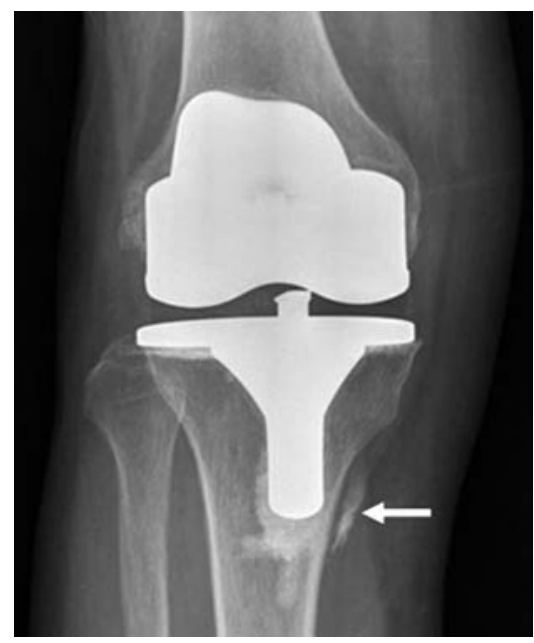

Fig. 3c

Radiographs showing a) contact between the medial tibial cortex and the cement mantle which is thinner at the medial side, b) that between the tibial stem and the medial tibial cortex and c) that between the tibial stem and the medial tibial cortex with leakage of cement (white arrow) outside the medial cortex beside the tip of the stem.

\section{Results}

The pre-operative radiological measurements showed that the mechanical axis was orientated in a varus direction and located medial to the axis of the tibial shaft in most knees. The mechanical axis was orientated in a varus direction in 175 knees $(71.1 \%)$ and located medial to the tibial shaft axis in 203 knees $(82.5 \%)$. The mean angle between the mechanical axis and the tibial shaft axis was $-0.7^{\circ}\left(-4^{\circ}\right.$ to $\left.+3^{\circ}\right)$. The mean distance of the mechanical axis from the tibial shaft axis was $-3.6 \mathrm{~mm}(-14$ to +7$)$.

The correlation coefficients of the associations of the demographic parameters showed that patient height was statistically significantly associated with the angle and distance between the mechanical axis and the tibial shaft axis (Pearson's correlation analysis, $\mathrm{r}=0.21, \mathrm{p}=0.001$ and $\mathrm{r}=0.17, \mathrm{p}=0.008$, respectively). There was a statistically significant correlation between the weight of the patient and the angle between the mechanical axis and the tibial shaft axis (Pearson's correlation analysis, $r=0.19$, $\mathrm{p}=0.003)$, but not with the distance between the mechanical axis and the axis of the shaft (Pearson's correlation analysis, $r=0.08, \mathrm{p}>0.05)$. Age, gender and BMI were not significantly correlated with the angle and distance between the mechanical and tibial shaft axes (Pearson's correlation analysis, $\mathrm{p}>0.05$ ).

Analysis of the post-operative radiographs showed that the mean distance of the tip of the stem from the tibial shaft axis was $-2.9 \mathrm{~mm}(-9$ to +4$)$. The tip was located medial to this axis in 196 knees $(79.7 \%)$. In 14 knees (5.7\%), radiological contact was found between the asymmetrical cement mantle surrounding the stem and the medial tibial cortex (Fig. 3a), and in two knees $(0.8 \%)$ direct radiological contact without an intervening cement mantle was found (Fig. 3b). One of these two knees showed leakage of cement medial to the medial tibial cortex (Fig. 3c). Clinical problems did not arise in any of these 16 patients. No component was found to have contact with the lateral cortex of the tibia.

Logistic regression analyses showed that radiological contact between the tibial stem and the cement mantle and the medial cortex of the tibia was statistically significantly associated with the pre-operative angle and the distance between the mechanical and tibial shaft axes (odds ratio (OR) 2.5, 95\% confidence interval (CI) 1.4 to 4.3 , $\mathrm{p}=0.001$ and OR $1.3,95 \%$ CI 1.1 to $1.5, \mathrm{p}=0.003$, respectively). Demographic factors (height, weight, BMI) and post-operative coronal and sagittal alignments of the tibial component did not have a statistically significant association with the radiological incidence of contact $(\mathrm{p}>0.05)$.

Comparisons between the Genesis II and the E-motion groups showed no significant differences in the preoperative parameters $(\mathrm{p}>0.05)$, but there were significant differences in the post-operative $(\mathrm{p}<0.05$; Table II). In the knees with the E-motion TKR, which has a generally greater medial offset throughout its size range (Table I) than that of Genesis II, the mean distance $(-3.8 \mathrm{~mm}(-8.8$ to 1.8$)$ vs $2.0 \mathrm{~mm}(-7.2$ to 4.2$)$, Student's $t$-test, $\mathrm{p}<0.001)$ of the tip of the stem medially from the tibial shaft axis and the proportion of the knees $(87.6 \%$ vs $72.0 \%$, chi-squared test, $\mathrm{p}=0.004)$ with the tip located medial to the tibial shaft axis were significantly greater than in knees with the Genesis II implant $(\mathrm{p}<0.05)$. These differences remained significant even after the values for the E-motion TKR were subtracted by $0.9 \mathrm{~mm}$, which was the mean difference in the pre-operative distance between the mechanical and tibial shaft axes. 
Table II. Comparison of the pre- and post-operative parameters in patients receiving Genesis II and E-motion total knee replacements

\begin{tabular}{|c|c|c|c|}
\hline Parameter & Genesis II (n = 125) & E-motion ( $n=121)$ & p-value \\
\hline \multicolumn{4}{|l|}{ Pre-operative } \\
\hline Mean $\left({ }^{\circ}\right)(\mathrm{SD})$ angle between the mechanical and tibial shaft axes ${ }^{*}$ & $-0.6(0.9)$ & $-0.7(0.9)$ & 0.499 \\
\hline Mean $(\mathrm{mm})(\mathrm{SD})$ distance between the mechanical and tibial shaft axes & $-3.2(3.9)$ & $-4.1(3.0)$ & 0.051 \\
\hline \multicolumn{4}{|l|}{ Post-operative } \\
\hline Mean $(\mathrm{mm})$ (SD) distance between the stem tip and tibial shaft axis ${ }^{\dagger}$ & $-2.0(2.4)$ & $3.8(2.5)$ & $<0.001$ \\
\hline $\begin{array}{l}\text { Adjusted number (\%) of knees with the tip located medial to the tibial shaft } \\
\text { axis }^{\ddagger}\end{array}$ & $90 \quad(72.0)$ & (84.3) & 0.021 \\
\hline Number $(\%)$ of the knees with contact between the stem and the tibia & $2(1.6)$ & $14 \quad(11.6)$ & 0.001 \\
\hline
\end{tabular}

* a negative value means that the mechanical axis is orientated to a varus direction to the tibial shift axis

$\dagger$ a negative value means that the mechanical axis is located medial to the tibial shaft axis or the tip of the stem

¥ since there was a difference with statistical tendency (Student's $t$-test, $p=0.51$ ) in the pre-operative distance between the mechanical

and tibial shaft axes, the values for the knees with an E-motion total knee replacement were subtracted by $0.9 \mathrm{~mm}$ which is the mean

difference of the pre-operative distance between the mechanical and tibial shaft axes from the original values

Knees with an E-motion TKR had a significantly higher proportion of the knee with contact between the stem or cement mantle and the medial cortex of the tibia $(11.6 \%$ vs $1.6 \%$, chi-squared test, $\mathrm{p}=0.001$ ).

\section{Discussion}

Most current TKRs have been developed using the anatomical data of normal knees of the Western population $7,13,14,20$ and therefore may not be suitable for Asian knees with different anatomical features. ${ }^{10,15,16}$ In our study, we attempted to determine whether a tibial component with a mediallyoffset stem would be a good option for Korean patients and found that in 16 knees $(6.5 \%)$ in our series, this design did not accommodate the anatomical features of the tibia in patients undergoing TKR for advanced OA.

In a study of ten Caucasian cadaver knees, Hicks et al, ${ }^{7}$ found that the tibial canal was positioned relatively medially in the tibial plateau, suggesting that the mechanical axis is typically located lateral to the axis of the tibial shaft. Based upon this finding, it was suggested that a mobile attachment of the stem on the tibial tray or an offset stem might be beneficial. Oswald et al, ${ }^{20}$ in a study of 44 Caucasian cadaver knees, found no significant differences between the mechanical axis and the anatomical axis of the tibia defined as the line connecting the midpoint of the tibial plateau and that of the tibial shaft $20 \mathrm{~cm}$ distal to the knee. By contrast, our study suggests that the mediallyoffset stem can present a problem in some Korean patients. In more than $80 \%$ of the knees, the mechanical axis was located medial to that of the tibial shaft and the mean distance of the mechanical axis from the tibial shaft axis medially was $3.6 \mathrm{~mm}$ (medially $14 \mathrm{~mm}$ to laterally $7 \mathrm{~mm}$ ). As expected from these pre-operative findings, the stem of the tibia was located medial to the tibial shaft axis in most $(79.6 \%)$ of our patients, and in 16 knees $(6.5 \%)$ this led to radiological contact between the stem itself or the cement mantle and the medial cortex of the tibia at TKR. In one knee with direct radiological contact, leakage of cement was found outside the medial cortex of the tibia, implying that the contact between the stem and the cortex had produced a fracture (Fig. 3c). Although none of the 16 knees with radiological contact have experienced clinical problems in the short term, the presence of an asymmetrical cement mantle may compromise the long-term fixation.

Our findings are in agreement with previous studies on Asian subjects. ${ }^{10,15}$ In a study of 72 Chinese patients undergoing TKR, the angle between the mechanical and tibial shaft axes was a mean of $1.8^{\circ}$ of varus indicating that the mechanical axis was located medial to the axis of the tibial shaft in many cases. ${ }^{15}$ A study of 133 Japanese patients with medial compartment OA showed that the mechanical axis was located medial to that of the tibial shaft and the authors warned of the possibility that the medially-offset stem of the tibial component might impinge against the medial cortex. ${ }^{10}$ Our study, taken together with these other studies on Asian knees, indicates that the medially-offset stem in a tibial component is not a better option for Asian knees.

Knees with an E-motion TKR had a greater mean distance of the tip of the stem medially from the tibial shaft axis and there was a greater number of knees with radiological contact between the tibial stem and the medial tibial cortex (Table II). This is to be expected since the E-motion system generally has a greater medial offset than the Genesis II system, particularly in the smaller components. However, we note that despite not applying any selection criteria to the allocation of prostheses, the mean preoperative distance of the mechanical axis medially from the axis of the tibial shaft was greater in the knees with the E-motion TKR (4.1 mm vs $3.2 \mathrm{~mm}$, Student's $t$-test, $\mathrm{p}=0.051$ ). Nevertheless, the difference in the mean postoperative distance remained significant even after adjustment by the pre-operative difference, suggesting that the knees receiving the E-motion device differed in their 
anatomical features from those with the Genesis II implant. Another confounding feature is that the E-motion system has a restriction in the pairing of the tibial component with a selected femoral component because of the high conformity between the articulating surfaces. Therefore, we may not have had the opportunity to select components one size smaller, which would have been possible with the Genesis II system. Furthermore, the larger diameter of the tibial stem in the E-motion system may have resulted in more cement being placed in the canal, which produced greater medial displacement of cement towards the medial tibial cortex. In order to exclude the apparent contact caused by a thick cement mantle, not caused by the design feature of the medial-offset stem, we counted only the knees in which the medial side of the cement mantle was thinner than the lateral side.

In our study, as in others, ${ }^{10,15,16,20}$ we have made twodimensional measurements from the radiographs. Detailed anatomical features of the tibia, the spatial relationship between the mechanical and tibial shaft axes, and the tibial stem would be better depicted by three-dimensional (3D) information. The proximal tibia has a triangular crosssection and some of the knees thought to show no radiological contact of the tibial stem to the tibial cortex may have had contact elsewhere depending on the extent of the posterior slope of the implanted tibial component. A future study obtaining 3D information would be justified.

Our study did not include data from replacements implanted with tibial components with different locations of stem. Therefore, future studies need to compare tibial components with medially-offset stems and those with no or laterally-offset stems.

Our study has shown that the design feature of the medially-offset stem in a tibial component may not be a good option in some Korean patients undergoing TKR for advanced $\mathrm{OA}$.

This study was sponsored by the research grant of the Korean Human Technical Engineering Research Fund (KOHTERF-2003-08).

No benefits in any form have been received or will be received from a commercial party related directly or indirectly to the subject of this article.

\section{References}

1. Brooks PJ, Walker PS, Scott RD. Tibial component fixation in deficient tibial bone stock. Clin Orthop 1984;184:302-8.

2. Ducheyne P, Kagan A 2nd, Lacey JA. Failure of total knee arthroplasty due to loosening and deformation of the tibial component. J Bone Joint Surg [Am] 1978;60A:384-91.

3. Fehring TK, Odum S, Griffin WL, Mason JB, Nadaud M. Early failures in total knee arthroplasty. Clin Orthop 2001;392:315-18.

4. Sharkey PF, Hozack WJ, Rothman RH, Shastri S, Jacoby SM. Why are total knee arthroplasties failing today? Clin Orthop 2002;404:7-13.

5. Cameron HU. Clinical and radiologic effects of diaphyseal stem extension in noncemented total knee replacement. Can J Surg 1995;38:45-50.

6. Pollice P, Lotke PA, Lonner JH. Principles of instrumentation and component alignment. In: Callaghan JJ, Rosenberg AG, Rubash HE, Simonian PT, Wickiewicz TL, eds. The adult knee. Vol. 2. Philadelphia: Lippincott Williams \& Wilkins, 2003:1085-93.

7. Hicks CA, Noble P, Tullos H. The anatomy of the tibia intramedullary canal. Clin Orthop 1995;321:111-16.

8. Lemaire P, Pioletti DP, Meyer FM, et al. Tibial component positioning in total knee arthroplasty: bone coverage and extensor apparatus alignment. Knee Surg Sports Traumatol Arthrosc 1997:5:251-7.

9. Hsu RW, Himeno S, Coventry MB, Chao EY. Normal axial alignment of the lower extremity and load-bearing distribution at the knee. Clin Orthop 1990;255:215-27.

10. Nagamine R, Miura H, Bravo CV, et al. Anatomic variations should be considered in total knee arthroplasty. J Orthop Sci2000;5:232-7.

11. Lotke PA, Ecker ML. Influence of positioning of prosthesis in total knee replacement. J Bone Joint Surg [Am] 1977;59-A:77-9.

12. Insall JN. Knee arthroplasty: limits and other problems: extensor mechanism complications. Orthopedics 1996;19:809-11.

13. Westrich GH, Haas SB, Insall JN, Frachie A. Resection specimen analysis of proximal tibial anatomy based on 100 total knee arthroplasty specimens. J Arthroplasty 1995;10:47-51.

14. Wevers HW, Simurda M, Griffin M, Tarrel J. Improved fit by asymmetric tibial prosthesis for total knee arthroplasty. Med Eng Phys 1994;16:297-300.

15. Ko PS, Tio MK, Ban CM, et al. Radiologic analysis of the tibial intramedullary canal in Chinese varus knees: implications in total knee arthroplasty. J Arthroplasty 2001;16:212-15

16. Matsuda S, Mizu-uchi H, Miura H, et al. Tibial shaft axis does not always serve as a correct coronal landmark in total knee arthroplasty for varus knees. J Arthroplasty 2003;18:56-62.

17. Tang WM, Zhu YH, Chiu KY. Axial alignment of the lower extremity in Chinese adults. J Bone Joint Surg [Am] 2000;82-A:1603-8.

18. Fleiss JL. Statistical methods for rates and proportions. Second ed. New York: John Wiley \& Sons Inc., 1981.

19. Shrout PE, Fleiss JL. Intraclass correlations: uses in assessing rater reliability. PSychological Bulletin 1979;86:420-8.

20. Oswald MH, Jakob RP, Schneider E, Hoogewoud HM. Radiological analysis of normal axial alignment of femur and tibia in view of total knee arthroplasty. J Arthroplasty 1993;8:419-26. 